\title{
Torsión, esfuerzos y deformaciones
}

\author{
Torsion, stresses and deformations
}

\author{
Yazmin E. Hernández-Sánchez ${ }^{a}$, Ana S. Salinas-Pérez ${ }^{b}$, Alberto Atilano-León ${ }^{c}$, José M. \\ Sandoval-Montaño ${ }^{d}$, Adolfo Briseño-Sánchez ${ }^{e}$
}

\begin{abstract}
:
In the work shown below, various research sources were analyzed in order to gather reliable information, in order to publicize the topic Torsion "stresses and deformations". The information that is presented is in its entirety true and concise, so the present theoretical basis is found in the results of this research presented. In this way, definitions, characteristics and applications in an industrial engineering environment are disclosed, both in turn in an approach to university students since the topics mentioned in this work are of importance and can be present throughout the industrial labor field, as it could be in a manufacturing or quality problem, for this the information here shows truthfully what the torsional moment of a cylindrical bar can be, examples of uniform and non-uniform torsion, independent axes at the moment that a force acts on them. This research provides accurate information on terms, applications and examples. This work was carried out with the aim of facilitating information to industrial engineering university students, hoping that this knowledge is of utmost importance in their future professional work life.
\end{abstract}

\section{Keywords:}

Torsion, stress, strain, axes

\section{Resumen:}

En el trabajo que se muestra a continuación, se analizaron diversas fuentes de investigación con la finalidad de recabar información confiable, para así dar a conocer el tema: Torsión "esfuerzos y deformaciones". La información que se presenta es en su totalidad veraz y concisa por lo que la presente base teórica se encuentra en los resultados de esta investigación presentada. De forma correcta se dan a conocer definiciones, características y aplicaciones en un entorno de ingeniería industrial, tanto a su vez en un enfoque a universitarios ya que los temas mencionados en este trabajo son de importancia y tienden a ser presentes a lo largo del campo laboral industrial, como lo pude ser en un problema de manufactura o bien de calidad; para ello la información aquí presentada demuestra con veracidad de lo que puede ser el momento torsor de una barra cilíndrica; ejemplos de torsión uniforme y no uniforme, ejes independientes al instante de que una fuerza actúa sobre ellos. En esta investigación se proporciona información certera de términos, aplicaciones y ejemplos. Este trabajo fue realizado con el objetivo de así facilitar la información a estudiantes universitarios de ingeniería industrial, deseando que este conocimiento sea de suma importancia en su futura vida profesional laboral.

\section{Palabras Clave:}

Torsión, tensión, deformación, ejes

\section{Introducción}

En algún punto de nuestra experiencia más de un individuo se ha preguntado qué sucede cuando comúnmente algún material cambia de forma, o ¿por qué cuando aplicamos alguna fuerza se llega a romper?, ¿por

a Autor de correspondencia, Universidad Autónoma del Estado de Hidalgo, https://orcid.org/0000-0002-2238-0847, Email: he419739@uaeh.edu.mx

b Universidad Autónoma del Estado de Hidalgo, https://orcid.org/0000-0002-1507-6687, Email: sa379958 @uaeh.edu.mx

c Universidad Autónoma del Estado de Hidalgo, https://orcid.org/0000-0002-2710-8033, Email: at419735@uaeh.edu.mx

d Universidad Autónoma del Estado de Hidalgo, https://orcid.org/0000-0002-5356-7006, Email: sa341210@uaeh.edu.mx

e Universidad Autónoma del Estado de Hidalgo, https://orcid.org/0000-0002-4732-4804, Email: br355010@uaeh.edu.mx 
qué razón algunas sustancias cambian de estado y otras no?, entre muchas otras cuestiones más. Por lo cual posteriormente será explicado de manera textual e ilustrativa la investigación previamente realizada.

A continuación, se explican los diferentes tipos de esfuerzos y deformaciones que presentan los materiales.

Torsión: Define a dos fuerzas aplicadas (figura 1), a algún objeto en diferentes direcciones dando como resultado un cambio físico sobre este o algún punto quiebre relacionado a las características del material que lo compone. [1]

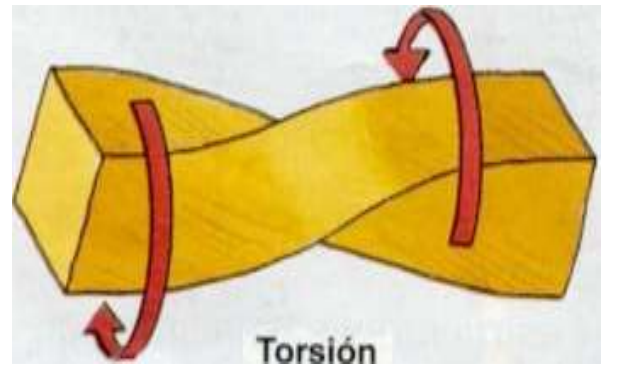

Figura 1. Torsión en una viga indicando el respectivo sentido del esfuerzo.

El concepto de esfuerzo se emplea con referencia al uso intenso de la fuerza (figura 2), el vigor o la mente para alcanzar un objetivo o superar determinados obstáculos. Por lo general, esforzarse resulta imprescindible para conseguir éxito. [1]

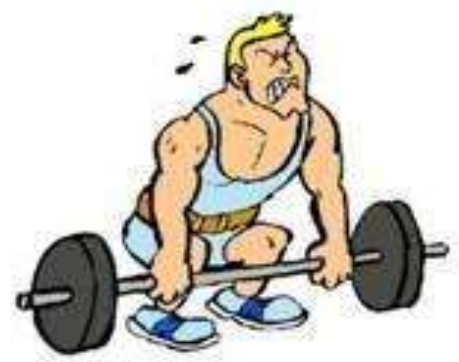

Figura 2. Esfuerzo requerido para levantar una pesa.

Es denominado deformación a el cambio realizado en un cuerpo (figura 3), que modifica características ya sea internas o externas de algún material o sustancia, algunos ejemplos de esos cambios podrían ser su tamaño y su forma. [2]

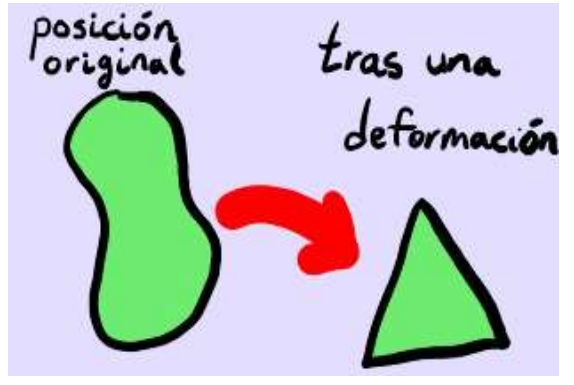

Figura 3. Ejemplo de deformación presente en alguna sustancia.

\section{Esfuerzo y deformación en barras cilíndricas}

Las barras cilíndricas son elementos de máquinas que transmiten a través de su contacto el movimiento circular entre sus dos ejes. [3]

Esfuerzo de corte desarrollado en un material sometido a un torque específico en un ensayo de $S=T . r / j$ donde: T= Es la torsión.

$r=E s$ la distancia desde el eje de giro a la fibra más exterior de la probeta.

$\mathrm{j}=$ Es el momento polar de inercia.

Se muestra el estado de esfuerzo de un elemento sobre algún eje sometido a flexión y torsión simultáneas; el círculo de Mohr correspondiente (figura 4). El esfuerzo cortante máximo $T_{\max }$ es igual al radio $R$ de la circunferencia y del triángulo rayado se obtiene: $\quad T=$ $R \sqrt{1 / 20}$. [3]

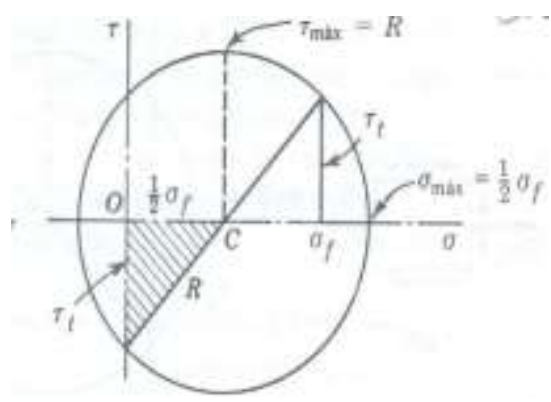

Figura 4. Círculo de Mohr presentando un boceto de magnitudes aplicadas, recuperado de: https://rb.gy/x7wajr

En alguna pieza prismática circular que es sometida a una torsión pura, bajo la acción de dos fuerzas en sentidos opuestos se pueden considerar algunos puntos los cuales nos aseguran una deformación por torsión los cuales son los siguientes puntos: 
- Las secciones están rectas y giran alrededor de su centro de gravedad.

- Las secciones rectas se conservan circulares y planas en la deformación.

- Los radios de la sección se conservan rectos.

- El ángulo entre dos radios permanece inalterado en la deformación.

En una pieza recta de sección circular sometida a torsión pura las de formación es tal que cada sección gira alrededor de su centro sin deformarse ni alabearse. [2.5]

\section{Esfuerzos y deformaciones en ejes independientes}

En los ejes independientes 0 de suspensión independiente, cada rueda está unida a un eje por separado (figura 5). Los vehículos de pasajeros modernos generalmente tienen este tipo de eje delante y detrás. [3]

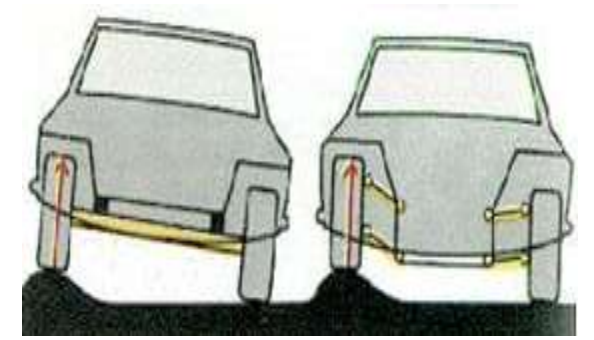

Figura 5 Ejemplo de ejes independientes al instante de aplicar alguna fuerza.

\section{Momento torsor}

Para determinar el momento torsor éste se realiza en el centro de las fuerzas dadas en la sección con el fin de ubicar el momento torsor real que actúa sobre ella. Al estudiar generalmente piezas prismáticas sometidas a un momento torsor, es algo muy complejo en las cuales se basa en la resistencia de materiales sobrepasando hipótesis fundamentales, avaladas únicamente con la experiencia. Esta complejidad consiste en que las partes sometidas a dicha torsión no permanecen planas, es decir, que sufren alguna deformación fuera de su mismo plano a lo cual se le conoce con el nombre de alabeo.

Alabeo, nos permite distinguir entre torsión uniforme y no uniforme, cuando el alabeo relativo es despreciable y no uniforme cuando el alabeo relativo es considerado.

(figura 6). [5]

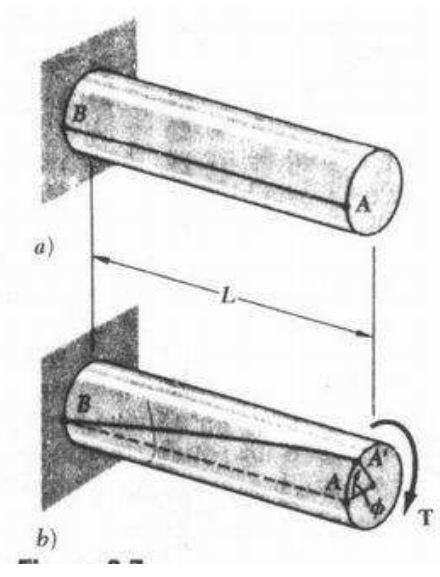

Figura 6. Ejemplo de torsión uniforme y torsión no uniforme en barras cilíndricas, recuperado de: https://rb.gy/ozlrkb

Se denomina momento torsor a la componente paralela al eje longitudinal del momento de fuerza resultante de una distribución de tensiones sobre una sección transversal del prisma mecánico. [4.5] Este instante puede aparecer cuando se someten estos elementos a la acción de un momento de fuerza o torque paralelo al eje del prisma, o cuando otro prisma mecánico perpendicular que está flexionado interseca al prisma mecánico original (figura 7). La relación entre el momento torsor y el campo de tensiones sobre la sección transversal de un prisma mecánico viene dada por: $M_{T} \int_{\Sigma}^{n} r \times t d y d z$ donde: $t=$ tensión tangencial, $r=$ el esfuerzo que se refiera a la relación entre el momento torsor y el campo de tensiones. [4-6]

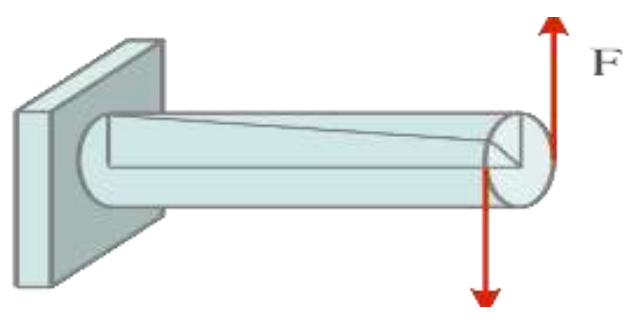

Figura 7. Momento torsor en una barra cilíndrica. 


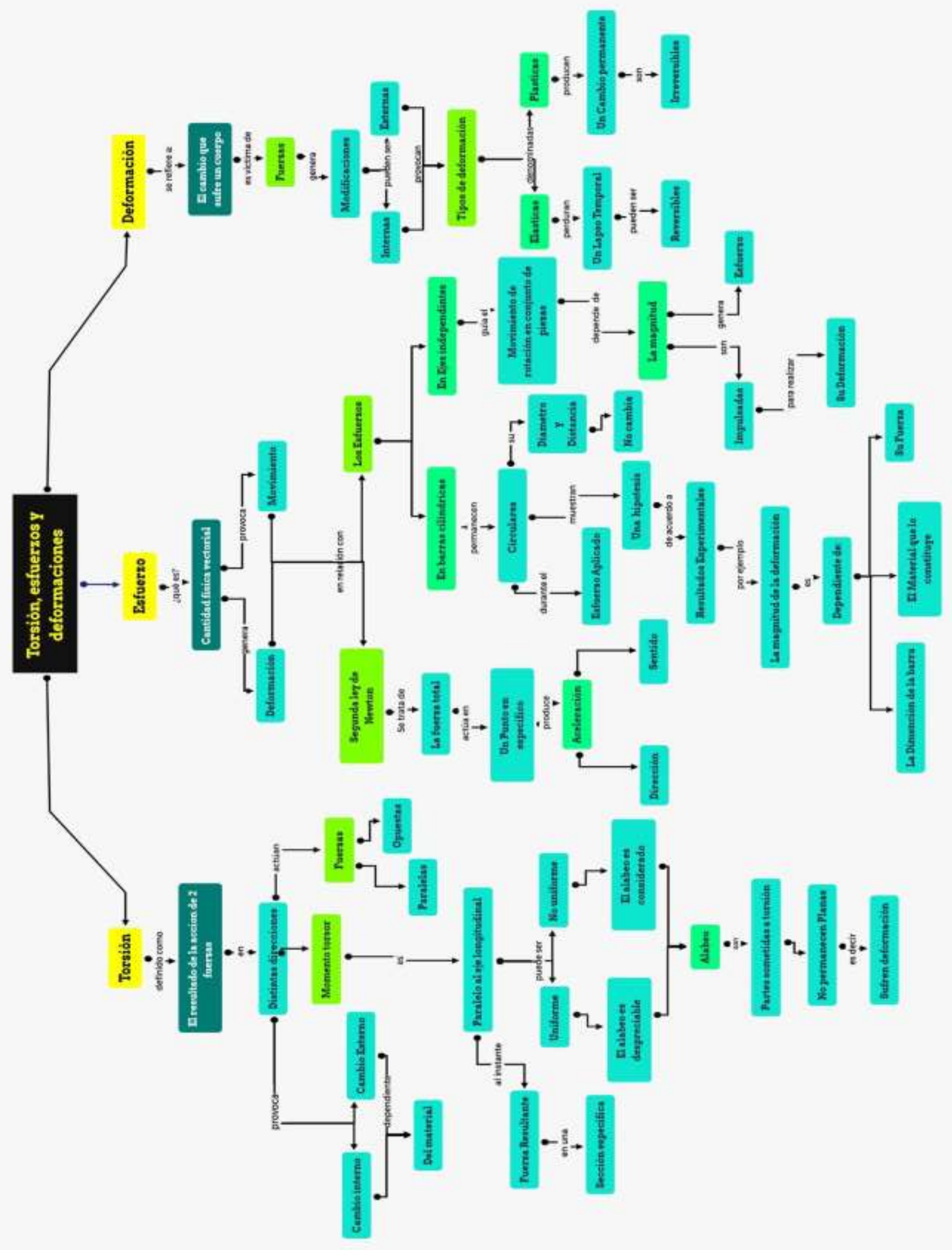




\section{Agradecimiento}

A nombre de nuestro equipo colaborativo queremos agradecer a la Dra. Lizeth Martínez Ayala, profesor investigador de la Universidad Autónoma del Estado de Hidalgo, campus Tepeji del Río, por su valiosa colaboración prestada para la revisión y aportación sobre el presente trabajo realizado.

\section{Referencias}

[1] Canet, J. M. (2012). Resistencia de Materiales y Estructuras. Barcelona: CIMNE. Obtenido de https://portal.camins.upc.edu/materials_guia/250120/2012/Resistencia $\% 20 \mathrm{de} \% 20$ materiales $\% 20 \mathrm{y} \% 20$ estructuras.pdf

[2] Chile, U. d. (2019). Obtenido de https://mecanicausach.mine.nu/media/uploads/deformaciones_axiales.pdf

[3] Luz Angela Silva Mesa, D. A. (Agosto de 2016). www.1library.com. Obtenido de https://1library.co/document/qo5ldx5y-banco-didacticopara-ensayo-de-torsion-en-barras.html

[4] M., A. M. (s.f.). Resistencia de Materiales Aplicada Primera Edición.

La Paz, Bolivia: Yucatán Hermosa. Obtenido de https://www.cartagena99.com/recursos/otros/apuntes/Resistencia_de_Mat eriales_Aplicada.pdf

[5] Timoshenco, S. (1957). Resistencia de Materiales. (T. D. Alba, Trad.) Madrid: Espasa Calpe S.A. Obtenido de http://cj000528.ferozo.com/cordoba/taller1/DEtaller1timoshendo.pdf

[6] Miguel Cervera Ruiz, E. B. (2001). Mecánica de Estructuras libro 1, Resistencia de materiales. (M. Andrew, Ed.) Jordi Girona Salgado 31,0834 Barcelona, España: Edicions UPC. Obtenido de http://cervera.rmee.upc.edu/libros/Mec\%C3\%A1nica_de_estructuras_I _Resistencia_de_Materiales.pdf. 